\title{
RISOTERAPIA: UNA TERAPIA COMPLEMENTARIA A LA MEDICINA OCCIDENTAL
}

\author{
JORGE LUCIANO VILLÁN GAONA ${ }^{1}$ \\ CIELO ESPERANZA GAONA ORDONEEZ ${ }^{2}$ \\ ZULI ADRIANA CARRERO GUTIÉRREZ ${ }^{3}$ \\ ${ }^{1}$ Médico y Cirujano, Universidad del Rosario, Bogotá, Colombia \\ ${ }^{2}$ Psicopedagoga, Secretaría de Educación Municipal de Cúcuta, Colombia \\ ${ }^{3}$ Médico y Cirujano, Universidad Militar Nueva Granada \\ Correspondencia: Jorge Luciano Villán Gaona \\ lucianovillangaona@gmail.com
}

Recibido: 18 de marzo de 2018 Aceptado: 5 de junio de 2018

\begin{abstract}
Resumen
La enfermedad y la hospitalización son factores estresantes, las instituciones tienen el deber de manejar de forma integral la salud de los pacientes. Para esto existen múltiples terapias complementarias como la risoterapia. Estas se basan en el humor para la promoción de la salud y se encargan de divertir a las personas, llevando sonrisas donde predominan los descontentos. Sus beneficios son múltiples y sus contraindicaciones son pocas, lo que la convierte en una estrategia costo-efectiva para mejorar la calidad de vida durante la hospitalización. Con este artículo, se pretende, no solo mostrar los asuntos más relevantes en la risoterapia, sino también motivar a investigar, así como concientizar al lector de la importancia de apoyar este nuevo boom.
\end{abstract}

Palabra clave: risoterapia; estrés; hospitalización; enfermedades crónicas; terapéutica

\section{LAUGHTER THERAPY: A COMPLEMENTARY THERAPY TO WESTERN MEDICINE}

\begin{abstract}
Disease and hospitalization are stressful factors, institutions have a duty to comprehensively manage patients' health. For this there are multiple complementary therapies such as laughter therapy. These are based on humor for health promotion and are responsible for entertaining people, bringing smiles where usually there is discontent. Its benefits are multiple and its contraindications are few, which makes it a cost-effective strategy to improve life quality during hospitalization. This article, aims not only to show the most relevant issues in laughter therapy, but also to motivate research, as well as to create awareness among readers of the importance of supporting this new boom.
\end{abstract}

Keyword: laughter therapy; stress; hospitalization; chronic diseases; therapy. 


\title{
RISOTERAPIA: UMA TERAPIA COMPLEMENTAR À MEDICINA OCIDENTAL
}

\begin{abstract}
Resumo
A doença e a hospitalização são fatores estressantes; nesse sentido, as instituições têm o dever de lidar de forma integral com a saúde dos pacientes. Para isso, existem múltiplas terapias complementares como a risoterapia. Esta se baseia no humor para a promoção da saúde e encarrega-se de divertir as pessoas, levando sorrisos aonde predominam os descontentamentos. Seus benefícios são múltiplos e seus contraindicações são poucas, o que a torna uma estratégia custo-efetiva para melhorar a qualidade de vida durante a hospitalização. Com este artigo, pretende-se não só mostrar os assuntos mais relevantes na risoterapia, mas também motivar a pesquisar, bem como conscientizar o leitor da importância de apoiar esse novo boom.
\end{abstract}

Palavra-chave: risoterapia; estresse; hospitalização; doenças crônicas; terapêutica.

Un corazón alegre es como una buena medicina pero un espíritu deprimido seca los huesos.

$\operatorname{Pr} 17,22$

\section{Introducción}

La Organización Mundial de la Salud (OMS) define salud como "un estado de completo bienestar físico, mental y social, no solamente corresponde a la ausencia de afecciones o enfermedades" (1). Esto conlleva la necesidad de un manejo integral de los pacientes, por lo que con el avance en la medicina y la tecnología no se debería permitir que algún paciente padezca de dolor, mucho menos niños con patologías oncológicas (2).

La enfermedad y la hospitalización suelen ser situaciones críticas con las que tienen que enfrentarse los niños alguna vez, que los convierten en sujetos susceptibles a factores estresantes en su vida $(5,6)$. Esto genera una angustia por buscar terapias complementarias, por lo que una alternativa es la risoterapia o geloterapia, del griego gelos, que significa reír (7), cuyo principal objetivo es enseñar a reír (2). En este sentido, es necesario integrarla en el tratamiento de pacientes pediátricos, con el propósito de suavizar el sufrimiento del niño y contribuir con todos los beneficios que se ha encontrado que aporta (6). La Resolución 5521 de 2013 habla de la psicoterapia como una intervención planificada y estructurada que tiene como objetivo influir sobre el comportamiento, el humor y los patrones emocionales de reacción a diversos estímulos (4).
Los que ejercen en el área de la salud están en riesgo de caer en una medicina deshumanizada que solo procura el abordaje de la enfermedad y no se preocupa por promover un mejor estado de bienestar, enfocándose en las dolencias del paciente pero con poca influencia en su bienestar (8).

Como afirma Gabriel García Márquez, "no hay medicina que cure lo que cura la felicidad" (3). El objetivo de esta revisión de tema es analizar los principales aspectos de la risoterapia, su importancia en la implementación como coadyuvante en el ambiente intrahospitalario, así como motivar al lector a investigar en esta temática y a que apoye los grupos que lideran esta iniciativa.

\section{Paciente hospitalizado}

Las enfermedades crónicas ocasionan que los pacientes sean sometidos a repetidas hospitalizaciones, quienes se enfrentan al temor o dolor de los procedimientos médicos, que generan alteraciones a nivel conductual, emocional y cognitivo en pacientes pediátricos. La mayoría de las investigaciones coinciden en que la hospitalización es una experiencia estresante y que obliga al paciente a enfrentarse a un ambiente que no le es propio (9-12).

Las personas sometidas a estrés continuo tienen una probabilidad mayor de sufrir diferentes disfunciones biológicas y son más propensas a sufrir enfermedades como hipertensión arterial, diabetes, enfermedad acidopéptica, cáncer, entre otras (10-14). 
Son múltiples los factores que influyen en la hospitalización, los cuales se pueden agrupar en tres grandes grupos: personales, familiares y hospitalarios, lo que indica la necesidad de un abordaje integral del paciente $(9,12)$.

Un estudio descriptivo transversal concluye que el factor más influyente en la percepción de estrés infantil es el daño corporal y la enfermedad, seguido del miedo a la muerte; sin embargo, la actitud que adoptan los padres es firme, quienes transmiten seguridad, al considerar que el beneficio para sus hijos es mayor pese al temor que lleva someterlos a una intervención médica (5).

Un estímulo doloroso pone en marcha potentes mecanismos y reacciones extremas o contradictorias, que pueden llevar desde la huida hasta la búsqueda desesperada por aliviar el dolor (3). Una alteración física por insignificante que sea en pacientes pediátricos puede repercutir de manera importante en la personalidad. De esta manera, los síntomas encontrados en enfermos pediátricos hospitalizados son alteraciones conductuales, déficits de atención, dificultad para la concentración, ansiedad y depresión, los cuales podrían tener un fuerte impacto psicológico, físico, social y ocupacional del paciente (9).

Como se puede dilucidar hasta aquí, la hospitalización, si bien es necesaria en muchos contextos, requiere estrategias para mejorar la calidad de vida del paciente hospitalizado.

\section{Estrategias complementarias en el paciente hospitalizados}

El estado psicológico puede afectar de manera positiva o negativa la evolución de la enfermedad $(9,14-15)$, por lo que se han buscado estrategias psicopedagógicas, nutricionales, corporales y espirituales de apoyo, que brindan una mejor calidad de vida a los pacientes; dentro de estas opciones se encuentra la risoterapia $(7,16)$. Cuando los pacientes usan el humor aligeran el ambiente difícil con su médico, y viceversa. Los profesionales de la salud que lo usan motivan a sus pacientes, con lo cual el humor y la risa se convierten en una herramienta valiosas que mejora la conexión entre cuidador, paciente y familia (17).

Dentro de los objetivo de estos programas están fomentar la actividad, proporcionar apoyo emocional, tratar de reducir las carencias escolares previniendo marginaciones, cultivar la alegría infantil y las relaciones sociales, fortalecer el carácter y la voluntad del niño, facilitar la adaptación a la hospitalización y a la enfermedad, mejorar la relación paciente-familiares y prestadores de salud, elementos que se suelen suspender en la hospitalización (9).
Un estudio aleatorizado mostró que la compañía de los padres y darles un juguete durante la premedicación disminuía la ansiedad y la aprensión producida en el momento de la premedicación oral (18).

Existen estrategias cognitivas enfocadas en la distracción para el control del dolor, sin embargo, solo logran la atención de la persona, de modo que es una alternativa si el dolor es de baja intensidad (19).

El humor va más allá de la simple distracción, al compararlo frente a otros métodos de distracción equiparados para el nivel de interés, se concluye que no es la cantidad de atención que demanda la actividad, sino el despertar emociones con la actividad lo que hace la diferencia (19).

Una investigación en niños concluyó que el grupo que fue acompañado por clowns durante la preanestesia presentó una disminución significativa de la ansiedad comparada con el grupo control (20).

Los padres y la risoterapia actúan sobre el estado de ánimo y el dolor que percibe el paciente, lo que la convierte en una terapia complementaria para una rápida mejoría, como se comprobó en un estudio realizado en dos niñas con cáncer sometidas a quimioterapia (21).

La necesidad de unión entre humanización y salud es cada vez más defendida, que se convierte en un fenómeno cultural posmoderno. Este hace énfasis en englobar cualquier forma de sufrimiento o dolor del individuo, y es aquí donde aparece el payaso hospitalario como efector de la risoterapia en tanto tratamiento complementario, que ayuda a controlar síntomas, a mejorar el bienestar y la calidad de vida de los paciente y de su entorno, sin reemplazar el tratamiento basado en la evidencia científica (16, 21-23). Evaluar el sentido del humor en los pacientes debería ser tan importante que ameritaría ser evaluado en el trabajo cotidiano del médico tratante (24).

\section{Clown hospitalario, humor, risa y risoterapia}

El clown hospitalario, denominado también payaso hospitalario o doctores clowns, son profesionales, artistas e intérpretes que tienen una capacitación adicional para trabajar con enfermos en el hospital, que se basan en el humor para la promoción de salud (22) y se encargan de divertir a las personas, llevando sonrisas donde predominan los descontentos $(2,16,24)$. Para desarrollar la risoterapia, deja la carpa convencional, y al usar la nariz roja, fluye la ingenuidad, el arte escénico y el humor, logrando generar empatía y emociones, recuperando la 
ternura y el contacto que se suele perder en el ambiente hospitalario, trasladando al público a un lugar agradable donde quisieran permanecer con ese amigo aliado que ofrece apoyo incondicional $(15,16,22,25-26)$.

El humor, definido como un estímulo que puede ayudar a las personas a reír y sentirse feliz (9), es una forma de comunicación destinada a causar diversión, una manera de producción simple de gestos (27), una cualidad que permite percibir la experiencia jocosa aun cuando las condiciones de la vida sean adversas (6) o simplemente es una interacción social que ocurre entre dos o más personas (24). Se habla del sentido del humor como un estado entre la alegría y la seriedad (24), es un rasgo psicológico que varía considerablemente y permite a las personas responder a diferentes tipos de estímulos (28). En el ser humano, el sentido del humor aparece alrededor de los 6 años (24), y entre los dos a cuatro meses aparece la sonrisa social, su ausencia o retraso se convierte en un indicativo de anormalidad en el neurodesarollo (29-30).

Risa y humor son una forma de afrontamiento instintivo que ayuda a enfrentar las decepciones y las luchas de la vida (31). Por medio de estos, se cree que las personas pueden reemplazar afectos negativos con positivos, ganan optimismo y esperanza de vida, de esta forma incrementa la habilidad de afrontar estados negativos, estresantes y amenazantes (32-33).

Sonrisa viene del latín subridere, que consiste en reírse levemente y sin ruido, es un efecto de humor, el más bello, armónico y saludable que se produce en el ser humano (34). La sonrisa aparece incluso a nivel intrauterino alrededor de la sexta y octava semana (16). El ser humano se comunica por medio del lenguaje verbal y no verbal. Al principio, los niños utilizan la risa como forma de comunicación, posteriormente se convierte en una conducta emocional. Aristóteles afirmaba que el bebé no es persona hasta que ríe. El ser humano deja de reírse de 400 veces al día a la edad de 6 años a tan solo 80 risas en los ancianos, de modo que en el adulto es de 15 a 100 risas por día. La filosofía tao recomienda reírse 30 veces al día. Las creencias hindúes aseguran que una hora de risa tiene efectos más beneficiosos en el cuerpo que cuatro horas de yoga (7).

La sonrisa de Duchenne involucra la contracción de los músculos cigomático mayor y menor, hay elevación de los labios, contracción de los orbiculares que eleva las mejillas y produce pliegues alrededor de los ojos; este tipo de sonrisa involucra emociones, es una risa genuina y espontánea (24, 35-36). Amand Duchenne, considerado como el padre de la neurología francesa, utilizaba la electricidad para explorar la expresión facial de las emociones y también como tratamiento; para Duchenne, cada músculo de la cara expresa una emoción; por ejemplo, el movimiento del músculo cigomático muestra satisfacción y alegría (36).

La risa es una respuesta psicofisiológica al humor o a cualquier estímulo con una secuencia de asociaciones neurofisiológicas, conlleva una contracción potente del diafragma con sonidos característicos producidos por la resonancia de la faringe, boca y cavidad nasal, por lo que produce una expresión facial típica dada por aproximadamente 50 músculos y mueve alrededor de 300 músculos en todo el cuerpo (28).

La risa es definida como una emoción ante diversos elementos intelectuales y afectivos, expresada de una forma ruidosa dependiendo del diafragma y de contracciones faciales o simplemente con un movimiento de la boca y otras partes del rostro que demuestra alegría (7); su opuesto es el llanto (17). Las funciones esenciales de la risa en el ser humano son unir, impulsar la salud y construcción de paz (32).

Superioridad, incongruidad, catarsis y play son las teorías que buscan explicar lo que ocasiona la risa (7). Está se puede clasificar según su etiología en cinco grandes grupos: la risa espontánea, simulada, estimulada, inducida o patológica (24). El humor es un estímulo y puede ocurrir sin la risa, y la risa es una respuesta y puede ocurrir sin humor $(24,35)$.

La risoterapia se define como el uso terapéutico de técnicas e intervenciones aplicables que conduce a un estado controlado de desinhibición, por lo que provoca risas $y$ emociones $(16,19)$. El mejor indicador inmediato de efectividad de esta es la risa, por esta razón para determinar si el humor será útil o no es indispensable conocer los principios de tiempo, receptividad y el contenido de la terapia. Así, se aconseja individualizar la terapia, teniendo precaución en pacientes en postoperatorio inmediato, glaucoma, ciertas enfermedades cardiorrespiratorias, psicóticos y agresivos; para la terapia, se debe evitar el humor destructivo, sexista, racial, crítico o a costa del paciente $(24,32)$. Efectos secundarios o adversos no tiene $(15,34)$; sin embargo, si un clown no sigue las reglas del hospital y sobrepasa los límites del paciente, puede tener efectos negativos (15); las bromas insensibles pueden resultar ofensivas e inquietantes (17). 
La risoterapia o geloterapia, interpretada principalmente por el clown hospitalario, es un complemento a las terapias tradicionales, que busca una mejor calidad de vida y mejorar la salud de los pacientes; no es un sustituto de los tratamientos convencionales y se puede aplicar en todas las edades $(7,33)$. La risoterapia consiste en reír de una manera natural, sana y que salga del vientre de un modo simple como en los niños; para que la risa sea efectiva y se pueda brindar sus innumerables beneficios, la carcajada debe ser franca y sincera (7). Fry en 1986 se refiere a la geloterapia como el estímulo al humor, con una respuesta emocional y conductual posterior (27).

La risa simulada logra una mayor intensidad y duración, ya que es por voluntad propia; a medida que se vuelve contagiosa, pasa a ser una risa espontánea, lo que traería mayores beneficios. En un estudio controlado en el que se estimulaba con una hora de risa una vez a la semana, se vio que la intervención tenía efectos positivos en la depresión, el insomnio y la calidad del sueño con respecto al grupo control (35).

Aún no está bien estipulado cómo debería realizarse la risoterapia, sin embargo, algunos autores hablan de tres fases: introducción, desarrollo y cierre (2). Si bien las rutinas son ensayadas, el juego que se desarrolla en cada cuarto depende mucho de cómo está el ambiente en cada habitación y de la necesidad específica de cada paciente; el tiempo promedio por cuarto es entre $30 \mathrm{y}$ 40 min (7). Para una adecuada praxis, se debe tener en cuenta el código deontológico Pupaclown (7).

\section{Risoterapia y salud}

La risoterapia no requiere mucho tiempo ni dinero, por lo que se podría implementar fácilmente, es una medida costo-efectiva (28), de modo que es beneficiosa para el paciente, su entorno y para el que la desarrolla; sin embargo, aún en nuestro país falta reconocimiento en este trabajo (13).

Esta terapia contribuye a que el paciente restaure el control y tenga una nueva perspectiva de su realidad. Para apreciar su aplicabilidad, se puede usar la teoría de los roles, en que el paciente desempeña un papel pasivo, sintiéndose indefenso al depender del médico para su tratamiento. Con el clown se busca agregar roles y descubrir antirroles; ejemplos de roles serían, payaso celoso, payaso derrotado, payaso amante cortejando, payado rebelde y luchador por la libertad, entre otros, que liberan la postura estática que tiene el paciente, los activa y busca un equilibro en el sistema de roles, ofreciendo una forma diferente de ver la realidad (25).
Laughter therapy, término ingresado desde hace poco como término mesh, sobre el cual hay pocos estudios con una adecuada metodología que evalué los beneficios, incluso muchos concluyen la necesidad de llevar investigaciones más rigurosas $(16,37)$.

Los beneficios de la risoterapia abarcan alrededor de 30 áreas de la salud $(18,35)$ y se ha descrito como una estrategia para mejorar la calidad de vida $(2,25,33)$ y como mecanismo protector para prevenir enfermedades y reducir el estrés (7, 24, 31). Para facilitar el entendimiento de las diferentes áreas, las agruparemos en efectos a nivel físico, cardiovascular, inmunológicos, endocrinológicos y psicosociales. $(2,6,7,15,19,22,24,25,27,32,35,38-40)$

\section{Efectos físicos}

Aumento de la tolerancia y reduce el dolor, disminuye la tensión muscular, con los movimientos musculares y del diafragma estimula los diferentes órganos.

Norman Cousins, en 1976, explica que diez minutos de risa genuina tras ver películas cómicas tuvo un considerable efecto analgésico y les dio por lo menos dos horas de sueño libre de dolor a pacientes con espondilitis anquilosante (28).

\section{Efectos cardiovasculares}

Aumenta el gasto cardiaco, la lipoproteína de alta densidad, disminuye la diferencia arteriovenosa de oxígeno y la resistencia vascular sistémica, de forma aguda incrementa la frecuencia cardiaca, el consumo de oxígeno y levemente la tensión arterial; variables que posteriormente disminuirán con la terapia. Inhibe el efecto del incremento de la glicemia posprandial, previene neuropatía diabética y las complicaciones microvasculares de la diabetes. Además, regula la expresión de genes involucrados en el ciclo celular y apoptosis.

Funciona en la rehabilitación cardiovascular produciendo menos arritmias, niveles de catecolaminas, y los pacientes suelen requerir menos $\beta$-bloqueadores y nitroglicerina (31), además mejora la función pulmonar en los fumadores (41).

\section{Efectos inmunológicos}

La principal rama encargada en este ámbito es la psiconeuroinmunología (7). Se ha descrito que mejora el sistema inmunitario aumentando citocinas antiinflamatorias, los linfocitos, las células $\mathrm{NK}$, el complemento C3, el interferón $\gamma$, y disminuye los niveles de citocinas proinflamatorias, hormona de crecimiento e IGF-2 e IL-6, reacciones alérgicas y también alérgenos específicos. 
Un estudio en el que veían videos de humor encontró que las células NK, inmunoglobulinas, complemento, leucocitos e interferón $\gamma$ permanecían elevados a las 12 horas de ver el video de humor, por lo que su uso diario podría prolongar la elevación de las células NK (31).

\section{Efectos endocrinológicos}

Aumenta la secreción de endorfinas, dopamina, oxitocina y serotonina, además estimula los receptores 5-hidroxitriptamina 1A. Disminuye los niveles de adrenalina y cortisol e inhibe la amígdala reduciendo la sensación de peligro.

\section{Efectos psicosociales}

Facilita la enseñanza, situaciones socialmente incómodas, la exteriorización de emociones y sentimientos, mejora las relaciones interpersonales, el autoestima, la comunicación, ayuda en la terapia del habla, aumenta la amabilidad, el optimismo, la esperanza, la creatividad e incrementa la actitud positiva, la confianza. Funciona como medida de afrontamiento, permitiendo aceptar de mejor manera la realidad, además disminuye la carga emocional, mejora la sensación de bienestar, el estado de ánimo del paciente. Sirve como un buffer para el estrés y el insomnio, disminuye enuresis, pesadillas, llanto, inapetencia y episodios de agitación.

\section{Neuroanatomía aplicada en la risoterapia}

El humor se produce en el lóbulo frontal $(7,9)$, sin embargo, la Society for Neuroscience ha ido al detalle al dividir la risa en tres áreas: corteza frontal, que es donde se entiende la situación humorística; la motora, que consiste en la corteza motora suplementaria que coordina los músculos para la risa; y el núcleo accumbens donde se centran las emociones y la felicidad $(14,17)$. La risa implica dos vías, ambas controladas en el puente troncoencefálico a nivel superior y dorsal: la vía voluntaria va desde el área premotora opercular dirigiéndose al tallo cerebral ventral, mientras que la vía involuntaria o emocional implica la amígdala, el tálamo, el hipotálamo, el subtálamo y el tronco cerebral dorsal $(14,17)$.

En un estudio en 22 niños de ambos sexos, en el que se les mostraban películas de humor, neutras o positivas mientras se le realizaba una Imagen por Resonancia Magnética nuclear se vio que las niñas preferían las de humor y los niños películas positivas. Las niñas estimulaban la corteza temporooccipital, mesencéfalo, amígdala con las películas de humor, en tanto que los niños el lóbulo bilateral parietal inferior, el giro fusiforme, el giro frontal inferior, la amígdala y la corteza prefrontal antero- medial con las películas positivas; esto es un gran indicio de que el humor es una emoción separada de la diversión regular y entre sexos podría haber diferencias (24).

\section{De la teoría a la práctica}

Tropa de Sonrisas de la Universidad Militar Nueva Granada fundada desde 2012 es un grupo integrado principalmente por estudiantes de Medicina, quienes dejan por instantes las batas blancas y el rol de médicos, para robar corazones, sonrisas y aplicar la risoterapia, fundamentalmente en pacientes pediátricos del Hospital Militar Central que padecen de alguna enfermedad oncológica.

En cada visita que realizan participan mínimo cuatro clowns acompañados de un "civil", persona encargada de la logística, de supervisar que no se cometan errores, entre otras funciones. En escenario o en los cuartos de los pacientes, suelen estar en parejas y por su paso, en mínimo veinticinco minutos, logran hacer más llevadera la realidad que padece el paciente y sus familiares.

Se considera que es necesario apoyar este tipo grupos e implementarlos como una terapia complementaria que permita el manejo integral de los pacientes. Asimismo, que pertenecer a un grupo como este permite fortalecer el sentido humano al atender a los pacientes; en cada visita quedan rostros de felicidad, muestra suficiente para continuar con la terapia que no solo beneficia al paciente, su entorno, sino también al que la practica.

\section{Conclusiones}

Hoy en día, hay una tendencia a caer en una medicina deshumanizada por diferentes factores, esto, asociado a todo lo que desencadena la estancia de hospitalización, obliga a los servicios de salud a enfocarse en una medicina integral. Para ello, es necesario que las instituciones de salud se familiaricen con las diferentes terapias alternativas, entre ellas, la risoterapia. Terapia costo-efectiva, fácil de aplicar enfocada en mejorar la calidad de vida tanto del paciente como de su entorno por medio de la risa, los estudios existentes muestran sus múltiples beneficios, pero es necesario ampliar la investigación y efectuar investigaciones con una estructura metodológica más sólida que permita sustentar su aplicabilidad. Por último, como medida a corto plazo, sugerimos apoyar a los grupos que lideran esta iniciativa y los invitamos a ser parte de este nuevo boom que busca en un principio aplicarse en espacios hospitalarios para después convertirse en un modelo de vida. 


\section{Agradecimientos}

Agradecemos a la Tropa de Sonrisas de la Universidad Militar Nueva Granada.

\section{Referencias}

1. Organización Mundial de la Salud. Conferencia Sanitaria Internacional. 2006. Disponible en: http://www.who.int/ governance/eb/who_constitution_sp.pdf.

2. Contreras MH, Garduño RJ, Carmona ADC, et al. Investigación materno infantil: la risoterapia como intervención de enfermería, para el control del dolor en niños en la aplicación de quimioterapia. Investig Matern Infant. 2011;3(3):128-31.

3. García, G. Del amor y otros demonios. Colombia: Alfred A. Knopf. 1994, 1:24.

4. Resolución 5521 de 2013. Ministerio de Salud y Protección Social. República de Colombia. 2013. Art 7.

5. Fernández-Castillo A, López-Naranjo I. Transmisión de emociones, miedo y estrés infantil por hospitalización. Int J Clin Heal Psychol. 2006;6(3):631-45.

6. Calmet Torres L, Regalado Benites M, Guevara Ángulo M. Influencia de la risoterapia en las características psicológicas y sociales del niño escolar hospitalizado. Rev Enferm Herediana. 2008;1(1):19-25.

7. Christian R, Ramos J, Susanibar, et al. Risoterapia: un nuevo campo para los profesionales de la salud. Rev Soc Per Med Inter. 2004;17(2):57-64.

8. Sanabria Ferrand PA. Reflexiones alrededor del concepto de promoción de la salud y prevención de la enfermedad. Revista Med. 2011;19(1):112-113.

9. Lizasoáin O, Ochoa B. Repercusiones de la hospitalización pediátrica en el niño enfermo. Osasunaz. 2003;5:75-85.

10. Sánchez Naranjo JC, Gutiérrez Segura JC, Santacruz Ibarra JJ, et al. El humor como estrategia terapéutica en niños hospitalizados en unidades pediátricas en Pereira (Colombia): reporte de una experiencia. Rev Colomb Psiquiat 2009;38(1):99-113.

11. Juárez MV, De la Cruz JA, Baena AJ. El estrés y las enfermedades crónicas. Medicina Familiar Andalucía. 2017;18(2):198-203.

12. Guevara-Gazca. M. P., Galan-Cuevas S. El papel del estrés y el aprendizaje de las enfermedades crónicas: hipertensión arterial y diabetes. Rev Latinoam Med Conduct. 2010;1(1):47-55.

13. National Institute of Mental Health. Las enfermedades crónicas y la salud mental: cómo reconocer y tratar la depresión [internet]. 2015 [citado 2019 mrz 14]. Disponible en: https://www.nimh.nih.gov/health/publications/espanol/ las-enfermedades-cronicas-y-la-salud-mental-como-reconocer-y-tratar-la-depresion/index.shtml

14. Gálvez JF. Trastornos por estrés y sus repercusiones neuropsicoendocrinológicas. Rev Colomb Psiquiatr. 2005;34(1):77-100.
15. Barkmann C, Siem A-K, Wessolowski N, Schulte-Markwort $\mathrm{M}$. Clowning as a supportive measure in paediatrics-a survey of clowns, parents and nursing staff. BMC Pediatrics. 2013;13(1):166. https://doi.org/10.1186/1471-243113-166

16. Lancheros García EA, Tovar Valle JF, Rojas Bermúdez CA. Risa y salud: abordajes terapéuticos. MedUNAB. 2011;14(1):69-75. DOI: https://doi org/10.29375/01237047.1380

17. Penson RT, Dignan FL, Canellos GP, et al. Burnout: caring for the caregivers. The Oncologist. 2000;5(5):425-434.

18. Golden L, Pagala M, Sukhavasi S, et al. Giving toys to children reduces their anxiety about receiving premedication for surgery. Anesth Analg. 2006;102(4):1070-2.

19. Weisenberg M, Tepper I, Schwarzwald J. Humor as a cognitive technique for increasing pain tolerance. PAIN. 1995;63(2):207-212. https://doi.org/10.1016/03043959(95)00046-U

20. Vagnoli L, Caprili S, Robiglio A, et al. Clown doctors as a treatment for preoperative anxiety in children: a randomized, prospective study. Pediatrics [internet]. 2005 oct [citado $2019 \mathrm{mrz}$ 14]; 116(4):563-567. Disponible en: www. pediatrics.org/cgi/doi/10.1542/\%5Cnpeds.2005-0466;

21. Alcocer Ballestas MC, Ballesteros, BP. Intervención "patch Adams" en indicadores de calidad de vida de dos niñas con cáncer [tesis de grado]. [Bogotá]: Pontificia Universidad Javeriana; 2005.

22. Espinosa BB, Gutiérrez TR. Lo esencial es invisible a los ojos: payasos que humanizan y promueven salud. Aletheia. 2010;31, 4-15.

23. Takeda M, Hashimoto R, Kudo T, et al. Laughter and humor as complementary and alternative medicines for dementia patients. BMC Complement Altern Med. 2010;10:28.

24. Woodbury-Fariña MA, Antongiorgi JL. Humor. Psychiatr Clin North Am. 2014;37(4):561-78. https://doi. org/10.1016/j.psc.2014.08.006

25. Grinberg Z, Pendzik S, Kowalsky R, Goshen Y. "Sancho." Drama therapy role theory as a context for understanding medical clowning. Arts Psychother. 2012;39(1):42-51. https://doi.org/10.1016/j.aip.2011.11.006

26. Reboredo CD. Los caminos del clown: Resistencia en movimiento. Juego, carnaval y frontera. Athenea Digit. 2011;11(2):157-71. https://doi.org/10.5565/rev/athenead/v11n2.675

27. Mallett J. Humour and laughter therapy. Complement Ther Nurs Midwifery. 1995;1(3):73-76.

28. Mora-Ripoll R. The therapeutic value of laughter in medicine. Altern Ther Health Med. 2010;16(6):56-64.

29. Sierra JC, Martín Ortiz, JD, Poveda Vera J. Efectos de una dosis de diazepam sobre el estado emocional en una muestra no clínica. Psicología y Salud. 2003;13(1):103-110.

30. Medina Alva MP, Caro Kahn I, Muñoz Huerta P, et al. Neurodesarrollo infantil: características normales y signos de alarma en el niño menor de cinco años. Rev Peru Med Exp Salud Publica. 2015;32(3):565-73. 
31. Balick MJ, Lee R. The role of laughter in traditional medicine and its relevance to the clinical setting: healing with ha! Altern Ther Health Med [internet]. 2003 jul-ag [citado 2019 mrz 14]; 9(4):88-91. Disponible en: http://citeseerx.ist.psu.edu/viewdoc/download?doi=10.1.1.522.67 $75 \&$ rep $=$ rep $1 \&$ type $=$ pdf

32. Wilkins J, Eisenbraun AJ. Humor theories and the physiological benefits of laughter. Adv Mind Body Med. 2009;23(6):8-12.

33. Villamil M, Quintero A, Henao E CJ. Terapia de la risa en un grupo de mujeres adultas. Fac Nac Salud Pública [internet]. 2013 may-ag [citado 2019 mrz 14];31(2):202208. Disponible en: http://www.scielo.org.co/pdf/rfnsp/ v31n2/v31n2a06.pdf

34. Hernández HMR. La sonrisa y su patología. Rev Mex Med Fis Rehab 2000;12(3):49-52.

35. Mora-Ripoll R. Potential health benefits of simulated laughter: a narrative review of the literature and recommendations for future research. Complement Ther Med. 2011;19(3):170-7. https://doi.org/10.1016/j. ctim.2011.05.003

36. Bourgeois ML. Guillaume Duchenne de Boulogne (18061875). Ann Médico-psychologiques, Rev Psychiatr. 2015;173(3):294-295. https://doi.org/10.1016/j. amp.2015.02.008
37. Meisel V, Chellew K, Ponsell E, et al. El efecto de los "payasos de hospital" en el malestar psicológico y las conductas desadaptativas de niños y niñas sometidos a cirugía menor. Psicothema. 2009;21(4):604-609.

38. Jaimes J, Claro A, Perea S, Jaimes E. La risa, un complemento esencial en la recuperación del paciente. Médicas UIS [internet]. 2011 abr [citado 2019 mrz 14];24(1):95100. Disponible en: https://revistas.uis.edu.co/index. $\mathrm{php} /$ revistamedicasuis/article/view/2584

39. Yun OB, Kim S-J, Jung D. Effects of a clown-nurse educational intervention on the reduction of postoperative anxiety and pain among preschool children and their accompanying parents in South Korea. J Pediatr Nurs. 2015;30(6):e89-e99. https://doi.org/10.1016/j. pedn.2015.03.003

40. Low L-F, Brodaty H, Goodenough B, et al. The Sydney Multisite Intervention of LaughterBosses and ElderClowns (SMILE) study: cluster randomised trial of humour therapy in nursing homes. BMJ Open. 2013;3(1):1-8. http:// dx.doi.org/10.1136/bmjopen-2012-002072

41. Raja A, Sundari FJ. Comparison of the efficacy of laughter therapy and breathing exercises on pulmonary function among smokers. Asian J Nurs Educ Res. 2014;4(1):105-10. 